\title{
Selective attention to the parts of an object
}

\author{
SHAUN P. VECERA \\ University of Iowa, Iowa City, Iowa \\ MARLENE BEHRMANN \\ Carnegie Mellon University, Pittsburgh, Pennsylvania \\ and \\ JOHN MCGOLDRICK \\ University of Denver, Denver, Colorado
}

\begin{abstract}
Recent empirical results suggest that there is a decrement in dividing attention between two objects in a scene compared with focusing attention on a single object. However, objects can be made of individual parts. Is there a decrement for dividing attention across different parts of a single object? We addressed this question in two experiments. In Experiment 1, we demonstrated that attention can exhibit part-based selection - that is, the subjects were more accurate in reporting two attributes from the same part of an object than they were in reporting attributes from different parts of an object. In Experiment 2 , we demonstrated that part-based attentional decrements occurred simultaneously with object-based attentional decrements. The results from Experiment 2 demonstrated that part-based attention is evident at the same time as objects are processed as coherent wholes. Our results imply that there is an attentional mechanism that can select either objects or their parts.
\end{abstract}

Findings in the visual attention literature indicate that when viewing a visual scene, humans can attend selectively not only to spatial regions (e.g., Eriksen \& Hoffman, 1973; Posner, 1980) but also to objects (e.g., Baylis \& Driver, 1993; Duncan, 1984; Egly, Driver, \& Rafal, 1994; Kramer \& Jacobson, 1991; Vecera, 1994; Vecera \& Farah, 1994). One of the earliest compelling demonstrations of this object-based selection came from Duncan (1984), who presented subjects with two spatially overlapping objects, a box and a line. The subjects were instructed to make decisions about two attributes present in the display; the two attributes belonged either to the same object or to the two different objects. The subjects were poorer at dividing attention between the two objects than they were at attending to a single object. This object advantage appeared as more accurate performance when they were reporting features of one object than when they were reporting features of two different objects. Furthermore, the subjects were able to identify two properties of a single object just as easily as one property of a single object (Duncan, 1984, 1993b).

Although Duncan's (1984) initial experiments have been criticized for several reasons (Baylis \& Driver, 1993; Watt, 1988), the within-object advantage is robust and has been replicated with several experimental paradigms (e.g., Behrmann, Zemel, \& Mozer, 1998; Duncan, 1993a;

The authors thank Don Hoffman, Maureen Marron, Philip Merikle, Mike Mozer, Hal Pashler, and two anonymous reviewers for comments on this research. Correspondence should be addressed to S. P. Vecera, Department of Psychology, 11 Seashore Hall E., University of Iowa, Iowa City, IA 52242-1407 (e-mail: shaun-vecera@uiowa.edu).
Egly et al., 1994; Kramer \& Jacobson, 1991; Kramer \& Watson, 1996; Lavie \& Driver, 1996; Vecera \& Farah, 1994). The results from these studies are consistent with the view that objects can be the target of selection.

Just as objects are part of a larger scene, objects themselves are made of parts, suggesting that the structure of a visual scene can be described at multiple levels (Navon, 1977; Palmer, 1977). For example, a finger may be represented as a whole object or as a part of a hand. This partwhole structure suggests that visual representations are hierarchical and may be decomposed iteratively into smaller elements. Theories of visual object recognition have postulated that objects are recognized by decomposing an object into its parts and the spatial relations among those parts (e.g., Biederman, 1987; Hoffman \& Richards, 1984; Hoffman \& Singh, 1997; Marr, 1982), even though these theories may not agree on what constitutes a part. What is common to these approaches is the notion that an input image is organized into parts at multiple spatial scales and that these parts play an important role in the structural description and representation of objects.

Because of the importance of parts in object recognition theories, a critical question for accounts of objectbased attention is whether parts of a single object can also be selectively attended. If parts of an object are integral to structured visual object representations (e.g., Biederman, 1987; Marr, 1982), the mechanisms of object-based attention might also apply at a more detailed level of decomposition into parts. If this were the case, we might expect to find an advantage for processing two features of a single part of an object compared with pro- 
cessing features from two different parts. This would demonstrate "part-based" attentional selection, analogous to object-based selection.

Before asking whether attention can be restricted to the parts of an object, we must first determine whether this issue has been addressed previously. Three lines of research indicate part-based selection at first glance. However, closer inspection shows that these lines of research do not demonstrate part-based selection. The first area of research involves global-local selection. Many studies have demonstrated that the subelements (possibly "parts") of a display can be attended and selected in hierarchical stimuli (e.g., a display consisting of the global letter $H$ made up of local letters, such as small $H \mathrm{~s}$ or $S \mathrm{~s}$; Navon, 1977). Selecting the local level of a hierarchical stimulus would correspond to part-based attention.

The second related area of research involves objectbased attentional selection. Demonstrations of objectbased attention might actually reflect part-based attention. For example, in the task used by Egly et al. (1994), the subjects detected targets that appeared at the end of one of two rectangles. The target was validly cued when a precue correctly predicted the target's location and was invalidly cued when a cue did not predict the target's location. When invalidly cued, the targets appeared in either the cued rectangle or in the uncued rectangle. The relevant result comes from the invalid trials; subjects are faster to respond to invalidly cued targets appearing in the cued object than in the uncued object. One could argue that the ends of the rectangles are different parts; thus, the difference between validly cued targets and the invalidly cued targets appearing in the cued rectangle could be due to part-based attention-that is, attention needs to shift from one part to another.

The third line of research involves part decomposition processes and the perception of symmetry and repetition. Driver and Baylis (1995) reported several findings in which subjects perceived the symmetry of two contours or the repetition of two contours more easily when the parts of those contours matched than when they mismatched. Contours containing matching parts may be more easily accessed by attention than contours containing mismatching parts, providing a mechanism of partbased attention.

The present research differs from these findings in important ways. First, the global-local results do not indicate whether all parts of an object are selected simultaneously, because all of the local parts (the small letters) are identical and are "wholes" (i.e., identifiable objects) in their own right. Second, the argument that the ends of the rectangles in Egly et al.'s (1994) task are separate parts is ad hoc. The rectangles likely would be perceived as single objects that do not have parts because there are no cues, such as minima of curvature (Hoffman \& Richards, 1984; Hoffman \& Singh, 1997) that would establish the ends as separate parts. Third, the symmetry and repetition perception studies (Driver \& Baylis, 1995) do not exam-
(A)

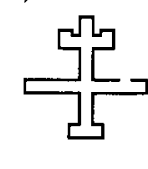

(B)

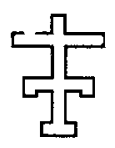

Figure 1. Examples of the stimuli used in Experiment 1. (A) Two stimulus objects; each contains two parts, a longer straight bar with a gap and a shorter bar with prongs. Half of the objects had the shorter bar above the longer bar (top) and half of the objects had the shorter bar below the longer bar (bottom). (B) The threshold-setting stimuli that consisted of the object's parts removed from the object context. (C) The pattern mask that followed the stimulus presentation.

ine whether there are costs in dividing attention across parts versus within a part.

In the present experiments, we asked whether parts of a single object could be selectively attended. We studied multi-part objects that were unlikely to involve spatial scaling effects, and the parts were defined empirically. To this end, we created a stimulus set and a task that permitted us to determine whether attention was limited in the number of object parts selected or whether attending to an object entailed selecting all of its parts. Examples of our objects (motivated by Tarr \& Pinker, 1989, 1990) appear in Figure 1A. The objects were created so that they would have parts defined by minima of curvature, a cue in which objects are divided into parts at the most concave points along an object (Hoffman \& Richards, 1984; Hoffman \& Singh, 1997). We verified empirically that observers would decompose these objects into parts at points of concavity. When asked to delineate the most natural parts of one of these shapes (Palmer, 1977), 12 naive subjects decomposed the stimuli into the main body and the two separate horizontal crossbars, consistent with parsing via minima of curvature.

Having verified that these objects were perceived as containing multiple parts, we used these objects in the selective/divided attention task developed by Duncan (1984) to investigate object-based attention. In our experiments, each part had two attributes. For example, one of the crossbars was short or long and had a gap on the left or right side; the other crossbar was short or long and had upward- or downward-turned prongs. The subjects were asked to report pairs of attributes; the attributes could come from either the same part or different parts. If attention can be directed to individual parts of a single object, attributes reported from a single part would be reported more accurately than parts reported from different parts. We tested this prediction in Experiment 1. 


\section{EXPERIMENT 1}

\section{Method}

Subjects. The subjects were 25 undergraduates who were each paid $\$ 5.00$ for their participation. All had normal or corrected vision.

Stimuli. The stimuli were small objects with a main body and base with two crossarms (Figure 1A). Only the two crossbars, one straight and one pronged, were relevant to the study. The straight crossbar was always longer than the pronged crossbar (even when the straight crossbars was in its shorter condition). The straight and pronged crossbar appeared equally in the upper and lower positions of the display.

The stimuli were viewed from a distance of $60 \mathrm{~cm}$. Each shape measured $1.8 \mathrm{~cm}$ tall $\left(1.72^{\circ}\right.$ of visual angle), with the straight and pronged bars yielding visual angles of $1.14^{\circ}$ and $0.66^{\circ}$ in their short positions and $1.52^{\circ}$ and $0.96^{\circ}$ in their long positions, respectively. The crossbars were located $0.4 \mathrm{~cm}$ from one another. The prongs on the shorter bar were oriented either upward or downward $0.3 \mathrm{~cm}$ above the height of the bar. The gap on the side of the straight bar was created by removing three pixels $0.4 \mathrm{~cm}$ from the left or right of the midline of the object. The pronged bar never contained the gap and the straight bar never contained prongs. The subjects were told to ignore the relative positions of the crossbars and to focus only on the attributes they were supposed to report. A pattern mask that measured $2.5 \mathrm{~cm}$ wide $\times 3.6 \mathrm{~cm}$ tall $\left(2.39^{\circ} \times 3.43^{\circ}\right)$ followed the stimulus.

Procedure. On each trial, the subjects' task consisted of reporting a pair of attributes. The attributes might both have come from the same crossbar (same part) or from different crossbars (different parts). There were only two possible same-part decisions. The subjects could report a straight bar length and gap (i.e., they had to decide whether the straight bar was long or short and whether the gap was on the left or right) or the subjects could report a pronged bar length and arms (i.e., they had to decide whether the pronged bar was long or short and whether the arms faced up or down). There were four possible different-parts decisions. (1) The subjects could report pronged bar length and straight bar gap, (2) straight bar length and pronged bar arms, (3) pronged bar length and straight bar length, or (4) pronged bar arms and straight bar gap. The subjects made all six judgments with judgment type held constant over a block of trials. The two attributes that the subjects were not to report in any particular block were held constant, and the subjects were aware of this. When not to be reported, the gap was held constant on the left side, the arms were held constant in the upward position, and both the shorter and longer crossbars were in the shorter rather than the longer condition (see Vecera \& Farah, 1994).

All stimuli were presented on a Macintosh Classic computer. Before the experimental trials, the subjects completed a threshold setting session to select an appropriate exposure duration. In this threshold session, the subjects were presented with two horizontal lines, one above the other, of the same lengths and relative spatial positions as those of the two crossbars of the experimental displays (Figure $1 B$ ). The subjects reported whether the longer of the two lines was on the top or bottom of the display. Each trial started with a ready signal of $800-\mathrm{msec}$ duration to prepare the subject. A central fixation followed for $800 \mathrm{msec}$. Thereafter, the stimulus appeared, centered over fixation, at some predetermined exposure duration. The pattern mask was then present for $200 \mathrm{msec}$. Following the mask, the screen was blank, during which time the subject responded. A delay of 1 sec preceded the next trial. The initial exposure duration was $150 \mathrm{msec}$ for each subject and was adjusted in $17-\mathrm{msec} \mathrm{dec}-$ rements until accuracy was between $70 \%$ and $85 \%$ correct on two consecutive blocks of 24 trials. This exposure duration was used for the experimental trials.

Following the threshold session, the experimental trials began. The timing was identical to the threshold session, except that the es- tablished exposure duration was used throughout the session. The mean exposure duration across subjects was $111 \mathrm{msec}(S E=$ $4.69 \mathrm{msec}$, range $100-133 \mathrm{msec}$ ). The subjects responded by using the left hand on the " 1 " and the " 2 " keys and the right hand on the " 9 " and the " 0 " keys on a standard keyboard. The subjects were asked to report the first attribute with their left hand and the second attribute with their right hand. The order of the attributes was counterbalanced across subjects, but for a given attribute, the response mapping remained the same. For example, half of the subjects were asked to report first which side the gap appeared on, whereas the other half were asked to report which side the gap appeared on second. However, the response mapping was identical so that subjects reported "left gap" by pressing the leftmost of the two keys (the "l" or the "9") and reported "right gap" by pressing the rightmost of the two keys (the "2" or the "0"). Similarly, "upward arms" was reported with the leftmost of the two keys, whereas "downward arms" was reported with the rightmost of the two keys. For the length dimension, short and long were reported with the leftmost and rightmost of the two keys, respectively.

Each subject completed eight blocks of trials, each containing 16 practice trials and 24 experimental trials for which responses were collected. Because the same-part condition had only two possibilities and the different-parts condition had four possibilities, the subjects performed each of the same-part reports twice so that we could equate the total number of trials between the same-part and differentparts conditions. Prior to each block, an instruction screen appeared informing the subjects which two attributes were to be reported in the following block and in what order the attributes should be reported. The order of the blocks was counterbalanced across subjects. The dependent measure was the percent of first and second attributes correctly reported on the same part and on different parts.

\section{Results and Discussion}

The data from 4 subjects were excluded because of near ceiling effects (average accuracy greater than $90 \%$ ). The accuracy data from the remaining 21 subjects were analyzed initially with a two-way analysis of variance (ANOVA) with part (same vs. different) and attribute order of report (first attribute reported vs. second attribute reported) as within-subjects factors. Figure 2 shows the mean accuracy rates.

The results suggested that dividing attention across parts resulted in performance decrements. There was a main effect of part, with accuracy being higher in the same-part condition $(80.06 \%)$ than in the different-parts condition $(75.10 \%)[F(1,20)=19.1, p<.003]$. There was, however, no main effect of order of report $[F(1,20)=$ $2.61, p>.10]$ with a nonsignificant difference between the first attribute reported $(78.81 \%)$ and the second attribute reported $(76.35 \%)$. There was also a significant interaction $[F(1,20)=6.15, p<.03]$; subjects' accuracy decreased when the second attribute was on a different part. This was confirmed by planned comparisons between the first and second attributes reported for the same-part condition and the different-parts condition. When the attributes were on the same part, the accuracy of the first attribute did not differ statistically from the accuracy of the second attribute $[t(20)<1]$. However, when the attributes were on different objects, the accuracy of the first attribute was significantly greater than the accuracy of the second attribute $[t(20)=5.32, p<.02]$. 


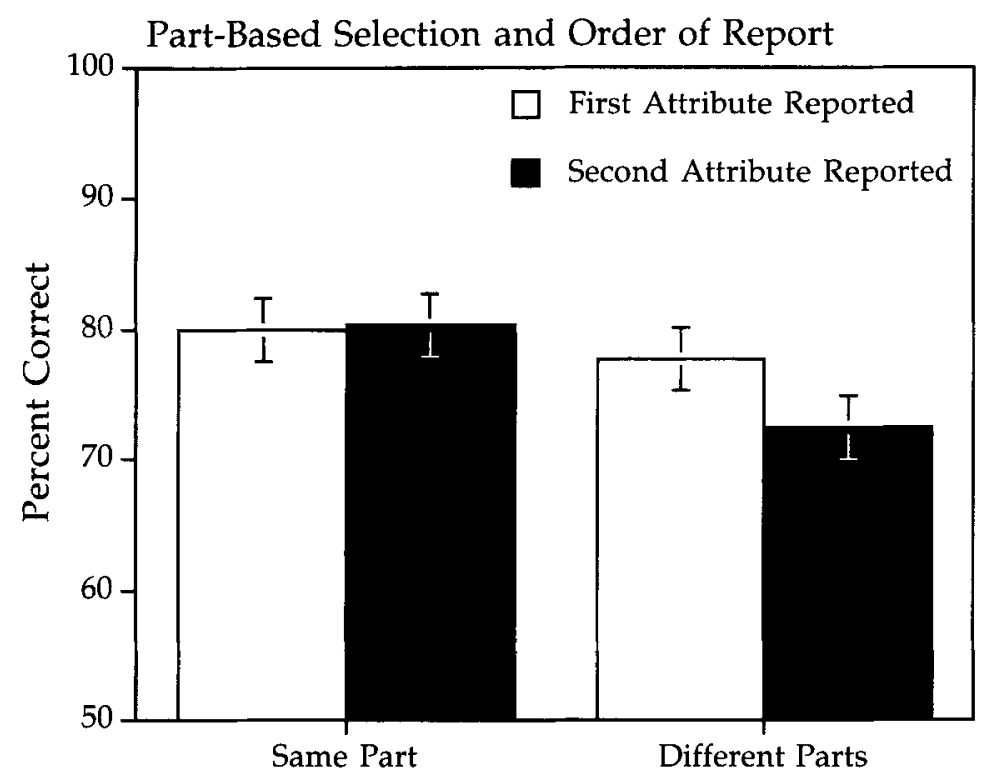

Attribute Location

Figure 2. Results from Experiment 1. Mean accuracy for reporting attributes located on the same or different parts by first attribute reported and second attribute reported. Error bars are $95 \%$ within-subject confidence intervals (Loftus \& Masson, 1994).

These results provide clear evidence that there was part-based attentional selection, which appears as a cost in selecting attributes from different parts of a single object. Two attributes located on a single part can be selected without cost; however, when two attributes are on different objects, a selection cost will be incurred. The accuracy decrease associated with report of the second attribute on a different part is analogous to Duncan's (1984) observation of the cost in switching from one object to a second. Thus, there is a single-part advantage analogous to the single-object advantage seen in Duncan's (1984) work. This part-based effect suggests that attention not only may be directed to single objects embedded in a multi-object scene, but also may be directed to individual parts of an object. Just as scenes are chunked into discrete objects, objects may be decomposed into discrete parts. Attentional selection presumably can occur from either level, object or part.

Although these results are straightforward, additional issues deserve discussion. First, in equating the number of trials in the same-part and different-parts conditions, we increased the subjects' exposure to the same-part condition, which may have contributed to the part-based effect. To examine this possibility, we reanalyzed our data from only the first half of the same-part trials and all of the different-parts trials. The results were similar to those reported previously. There was a main effect of part $[F(1,20)=5.01, p<.05]$ and a marginal main effect of order of report $[F(1,20)=4.25, p<.06]$. There was also a nonsignificant interaction $[F(1,20)=1.37, p<.30]$. This nonsignificant interaction probably was caused by reduced statistical power due to the removal of one quarter of the trials. The critical result is the presence of a main effect of part, which remained statistically significant with the reduced statistical power. The same-part advantage was not merely a consequence of increased experience with the same-part condition.

The second issue to discuss concerns subjects' accuracy in reporting the four different attributes in our stimuli. Was the part-based effect the result of one or two of the attributes, or was this effect observed in all of the attributes? To examine the accuracy for same and different parts for each of the four attributes separately, we performed a two-way ANOVA on accuracy, with attributes (straight bar length, pronged bar length, gap side, and prong direction) and condition (same part/different parts) as within-subjects factors. The data were collapsed across order of report (first vs. second attribute reported). The main effect of part was replicated, with higher accuracy for attributes on the same part $(80 \%)$ than on different parts $(74.95 \%)[F(1,20)=19.9, p<.0002]$; when all four attributes were averaged together, accuracy was greater in the same-part condition than in the different-parts condition. There was also an effect of attribute $[F(1,20)=$ $13.91, p<.0001]$, indicating that the different attributes differed from one another. The subjects were most accurate in reporting the prong direction $(86.55 \%)$, followed by reporting side of gap (79.16\%), then by the length of the longer crossbar $(73.61 \%)$, and then by the length of the small bar $(70.58 \%)$. Finally, there was a two-way interaction between part and attribute $[F(3,60)=3.88$, $p<.02]$, indicating that the accuracy of reporting attributes 
Table 1

\begin{tabular}{|c|c|c|c|}
\hline \multicolumn{4}{|c|}{$\begin{array}{l}\text { Mean Percent Correct for Attributes and } \\
\text { Same-Part and Different-Parts Conditions }\end{array}$} \\
\hline \multirow[b]{2}{*}{ Attribute } & \multicolumn{2}{|c|}{ Condition } & \multirow[b]{2}{*}{$\begin{array}{c}\text { Difference } \\
\text { (Same - Different) }\end{array}$} \\
\hline & $\begin{array}{c}\text { Same } \\
\text { Part }\end{array}$ & $\begin{array}{l}\text { Different } \\
\text { Parts }\end{array}$ & \\
\hline Prongs (up/down) & 88.78 & 84.32 & $4.46^{*}$ \\
\hline Gap (left/right) & 84.11 & 74.2 & $9.91 \dagger$ \\
\hline Length of shorter bar (short/long) & 74.3 & 66.87 & $7.43^{*}$ \\
\hline Length of longer bar (short/long) & 72.82 & 74.4 & -1.58 \\
\hline
\end{tabular}

Note-The $95 \%$ within-subject confidence interval is the condition mean \pm 3.56 . ${ }^{*} p<.05 . \quad{ }^{+} p<.001$.

differed between the same-part and different-parts conditions. Table 1 shows the means, $95 \%$ confidence intervals, and $p$ values for this comparison. Three of the four attributes were reported more accurately in the same-part condition than in the different-parts condition. The fourth attribute, the length of the longer bar, was reported slightly more accurately in the different-parts condition than in the same-part condition, although this difference was not statistically significant. Thus, our findings generalize across the attributes used in our stimuli.

The third and most substantial issue for discussion concerns whether our demonstration of part-based attention could have been due to the task used. Our task emphasized the coding of separate parts, which may have biased the subjects to treat each stimulus not as an object, but as a conglomeration of parts. Thus, the part-based effects we have reported may occur only with stimuli in which subjects preferentially attend to parts, which may prevent subjects from representing the objects created by the parts. In Experiment 2, we addressed this issue by using two-object displays. The subjects reported two attributes from these displays. To determine whether our stimuli are processed as single objects, we included a condition to assess any object-based costs of attentional selection. When the subjects reported pairs of attributes, those attributes could have occurred on the same part or on different parts, and the attributes could also have occurred within a single object or within different objects. Because the displays contained two objects, we should be able to replicate the standard object-based attention effect in which attributes are reported more accurately from the same object than from different objects. Furthermore, if our results were due to attentional selection at different levels of the object-part hierarchy, we also should be able to replicate the part-based effect observed in Experiment 1. However, if the results of Experiment 1 were due to an idiosyncratic part decomposition of the stimuli, we would not be able to replicate the part-based effect.

\section{EXPERIMENT 2}

\author{
Method \\ Subjects. The subjects were 40 undergraduates who participated \\ in the experiment for course credit. All had normal or corrected vi- \\ sion; none had participated in the previous experiment.
}

Stimuli. The stimuli were identical to those used in Experiment 1, except that two shapes were presented simultaneously. The two shapes appeared side by side, located $1.6 \mathrm{~cm}$ from one another measured from the centers of the main bodies. The overall display measured $4.9 \mathrm{~cm}$ wide $\left(4.67^{\circ}\right)$ and $1.8 \mathrm{~cm}$ tall $\left(1.72^{\circ}\right)$. Two small arrows, one red and one green, each $1 \mathrm{~cm}$ in length and located $0.3 \mathrm{~cm}$ from the outside edge of the crossbar, also appeared together with the stimuli. The arrows indicated which attributes were to be reported.

The single objects used previously were paired with each other and with themselves. Half of the time any given shape appeared on the left, and half of the time the same shape appeared on the right. In the two-object displays, the shorter (pronged) bar appeared in the upper position (i.e., above the longer bar) on both objects on one quarter of the trials; similarly, the longer bar appeared in the upper position on both of the objects on one quarter of the trials. In the remaining half of the trials, the part order differed between the two objects (i.e., the shorter bar was in the upper position on one object and in the lower position in the other object). There were 42 different displays.

The experimental design involved factorially crossing the part and object factors. The attributes reported could be located on the same part and on the same object, on different parts of the same object, on the same part but on different objects, or on different parts of different objects. When the attributes were on different objects, same part and different parts were determined on the basis of whether the attributes were on corresponding parts (i.e., if both attributes were on the shorter, pronged bar, the parts were to be reported the same because they corresponded to one another; if one attribute was on the pronged bar and the other was on the straight bar, the parts were to be reported different).

Procedure. The procedure was identical to that in Experiment 1 , with the following exceptions: In the threshold setting session, the subjects saw displays containing two sets of two horizontal lines, one set on the left and one set on the right. This display approximated the arrangement of the two bars of each of the two objects in the experimental displays. The subjects reported attributes from "parts" that did not appear in an object (i.e., without the objects' central body).

Because of the larger number of displays in this study, each subject reported only a subset of the part attributes. The subjects in one group reported the length of one of the two parts and prong direction, and the subjects in the other group reported the length of one of the two parts and gap side. The order of report was counterbalanced in each group. This procedure allowed us to manipulate whether the attributes were located on the same part or on different parts without requiring all part pairings to be used (which could have caused subject fatigue, because of the large number of trials).

Because any given display would contain multiple parts and attributes, we needed to indicate which attributes should be reported. We used small colored arrows as indicators. The crossbar for which length was to be reported was indicated by a red arrow; the gap side or prong direction was indicated by a green arrow. On each trial, the subjects reported their two attributes (e.g., prong direction and line 
length), and these attributes could appear on the same part or on two different parts. For example, if a subject reported the prong direction and the length of the pronged part, the attributes were located on the same part. If a subject reported the prong direction and the length of the straight part, the attributes were from different parts. When the arrows pointed at different parts, they could be the two parts above each other (different parts/same object), two parts beside each other (same or different parts/different objects), or two lines on the diagonal from each other (same part or different parts/ different objects)

The timing of the display and mask and the method for determining threshold were identical to those in Experiment 1, except that the starting exposure duration was $350 \mathrm{msec}$. The exposure duration was increased or decreased in $35-\mathrm{msec}$ intervals. The subjects' accuracy was set at approximately $70 \%$. The exposure duration was then kept constant throughout the experimental session. The mean exposure duration across all 40 subjects was $294 \mathrm{msec}(S E=$ $5.06 \mathrm{msec}$; range, $250-350 \mathrm{msec}$ ).

The experimental session was identical to the threshold session, except that the full objects were presented. For a single subject, the two attributes reported in the threshold session were reported in the experimental session. The subjects responded by using the " 1 " and " 2 " keys for the first report and the " 9 " and " 0 " keys for the second report. The subjects completed 12 practice trials drawn randomly from the four conditions before beginning the experimental trials. The order of attribute report was counterbalanced. The crossing of part and object was a within-subjects factor. Accuracy was emphasized over speed. The subjects completed four experimental blocks of 84 trials each with a rest between blocks.

\section{Results and Discussion}

None of the subjects were excluded from the analysis. A three-factor within-subjects ANOVA was performed with part (same vs. different), object (same vs. different), and report (first vs. second) as factors. Figure 3 depicts the part and object effects. Importantly, there were main effects for part $[F(1,39)=16.76, p<.0002]$ and for object $[F(1,39)=61.45, p<.0001]$. The subjects were more accurate in the same-part condition $(84.03 \%)$ than in the different-parts condition $(79.25 \%)$; the subjects were more accurate in the same-object condition (85.63\%) than in the different-objects condition $(77.67 \%)$. There also was a main effect for report $[F(1,39)=6.27, p<.01]$. There were two-way interactions between part and object $[F(1,39)=5.20, p<.03]$ and between object and report $[F(1,39)=10.35, p<.003]$, the latter replicating Duncan's (1984) results. The interaction between part and report showed the same pattern as in Experiment 1, but did not reach statistical significance $[F(1,39)=1.12$, $p<.30]$. The three-way interaction was not significant $[F(1,39)<1]$.

Two theoretically important findings emerged from this experiment. First, we replicated the part-based effect by using multiple object displays. Second, in addition to the part effect, an object effect emerged as well. Thus, when the subjects did not know they were to attend to the global object or to the local part, part and object effects occurred simultaneously. Object-based processing occurred within the displays, and part-based processing occurred while the objects were processed as discrete "wholes" (see also Driver \& Baylis, 1995). Part-based selection does not appear to be caused by idiosyncratic part decomposition in our stimuli.

Our results also replicated the interaction between object and order of report observed by Duncan (1984); there was a strong trend toward an interaction between part

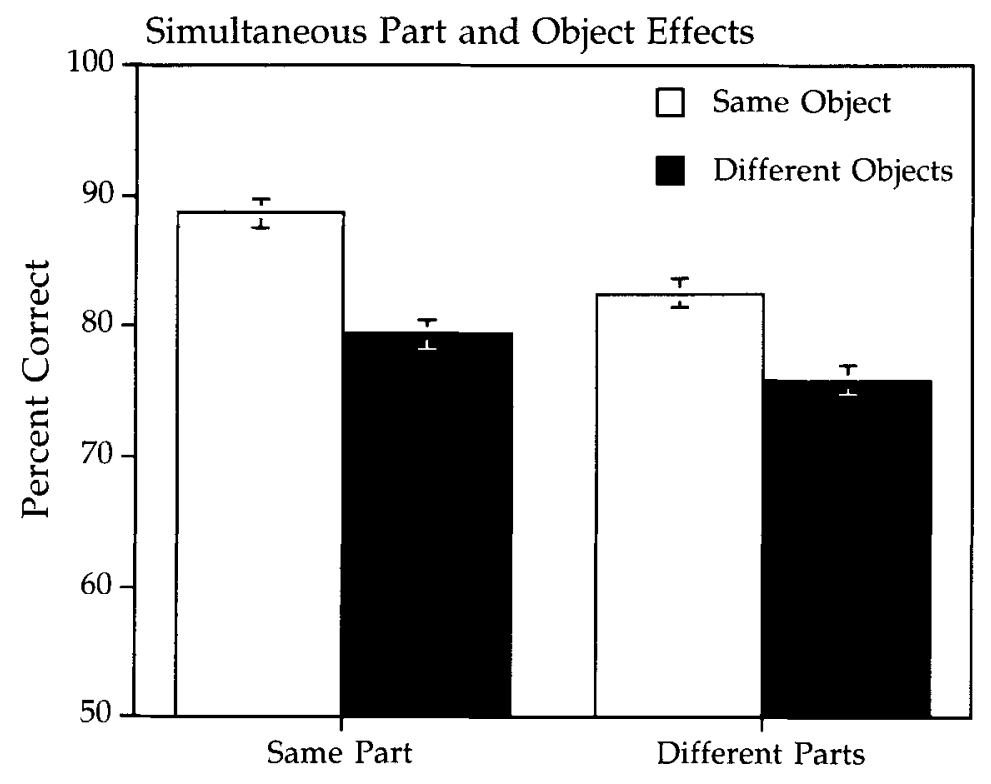

Attribute Location

Figure 3. Results from Experiment 2. Mean accuracy for reporting attributes located on the same or different parts and on the same or different objects. Error bars are $95 \%$ within-subject confidence intervals. 
and order of report, consistent with our results from Experiment 1 . Finally, the interaction between part and object indicates that the part-based effect is smaller when the attributes are on different objects. Planned comparisons revealed significant part-based effects when the attributes were on the same object $[t(39)=3.91, p<.0004]$, replicating Experiment 1 , and also when the attributes were on different objects $[t(39)=3.54, p<.002]$. Although the part effect was smaller when the attributes were on different objects, this effect remains statistically significant.

One could argue that we may not have demonstrated object-based effect because the objects were separated in space. The difference in reporting attributes from the same object and from different objects might have been due to spatial attention. However, the distance between the objects was relatively small (i.e., $1.52^{\circ}$ ), which would have discouraged the use of spatial attention (Duncan, 1984); other empirical results also indicate that spatial attention may not have been involved in this task (Vecera, Behrmann, \& Filapek, in press).

\section{GENERAL DISCUSSION}

In two experiments, we demonstrated that dividing attention between parts is more difficult than attending to a single part, resulting in part-based visual attention. This single-part advantage also appears in multi-object displays in which object-based attentional effects are observed, indicating that both part-based and object-based attentional limitations can be observed concurrently.

Our results are compatible with theories of object recognition that hold that part decomposition is required for recognition (Biederman, 1987; Marr, 1982). Part decomposition appears to influence the allocation of attention within an object. Thus, part decomposition may occur in both object recognition and object-based visual attention. Indeed, part decomposition in object recognition may occur by virtue of object-based and part-based visual attention. We view the enhancement of objects and parts of objects as part of the recognition process. Our demonstration of a part-based attentional cost may serve to integrate the object recognition literature with the objectbased attention literature, two fields that have tended to remain separate from one another. Future studies will be required in order to explore the relationship between object recognition and object-based attention.

Finally, we must address the locus of part-based selection. Does this form of selection occur earlier or later in the visual processing stream? On the basis of our findings, we are agnostic as to the visual representation from which selection occurs in this paradigm. On the one hand, because the attributes may be coded with respect to the part or object (e.g., the gap is coded to the left or right of the crossbar), selection could occur from a late, object-based representation (or structural description) that codes parts relative to an object's geometry in a non- spatial format. On the other hand, selection could be mediated through an array format (or spatiotopic) representation, in which the parts are coded in terms of their spatial locations; the parts could be delimited from one another by stimulus level cues, such as concave cusps or minima of curvature (Braunstein, Hoffman, \& Saidpour, 1989; Gibson, 1994; Hoffman \& Richards, 1984; Hoffman \& Singh, 1997; Stevens \& Brooks, 1988). The representation that is selected from is likely to be influenced by many factors, including the task that subjects perform (Vecera \& Farah, 1994), so an "either-or" answer may not be possible. This conclusion, however, does not detract from our major finding and conclusion that visual attention exhibits a limitation in the number of parts that can be selected concurrently.

\section{RËFERENCES}

BAYLIS, G. C., \& DRIVER, J. (1993). Visual attention and objects: Evidence for hierarchical coding of location. Journal of Experimental Psychology: Human Perception \& Performance, 19, 451-470.

BehrmanN, M., Zemel, R., \& Mozer, M. C. (1998). Object-based attention and occlusion: Evidence from normal subjects and a computational model. Journal of Experimental Psychology: Human Perception \& Performance, 24, $1011-1036$.

BIEDERMAN, I. (1987). Recognition-by-components: A theory of human image understanding. Psychological Review, 94, 115-147.

Braunstein, M. L., Hoffman, D. D., \& Saidpour, A. (1989). Parts of visual objects: An experimental test of the minima rule. Perception, 18, 817-826.

DRIVER, J., \& BaYlis, G. C. (1995). One-sided edge assignment in vision: 2. Part decomposition, shape description, and attention to objects. Current Directions in Psychological Science, 4, 201-206.

DunCAN, J. (1984). Selective attention and the organization of visual information. Journal of Experimental Psychology: General, 113, 501. 517.

DUNCAN, J. (1993a). Coordination of what and where in visual attention. Perception, 22, 1261-1270.

DunCaN, J. (1993b). Similarity between concurrent visual discriminations: Dimensions and objects. Perception \& Psychophysics, 54, 425-430.

Egly, R., Driver, J., \& RAFAL, R. D. (1994). Shifting visual attention between objects and locations: Evidence from normal and parietal lesion subjects. Journal of Experimental Psychology: General, 123, 161-177.

ERIKSEN, C. W., \& Hoffman, J. E. (1973). The extent of processing of noise elements during selective encoding from visual displays. Perception \& Psychophysics, 14, 155-160.

GiBSON, B. S. (1994). Visual attention and objects: One versus two or convex versus concave? Journal of Experimental Psychology: Human Perception \& Performance, 20, 203-207.

Hoffman, D. D., \& Richards, W. A. (1984). Parts of recognition. Cognition, 18, 65-96.

Hoffman, D. D., \& SinGH, M. (1997). Salience of visual parts. Cognition, 63, 29-78.

KRAMER, A. F., \& JaCoBson, A. (1991). Perceptual organization and focussed attention: The role of objects and proximity in visual processing. Perception \& Psychophysics, 50, 267-284.

Kramer, A. F., \& Watson, S. E. (1996). Object-based visual selection and the principles of uniform connectedness. In A. F. Kramer, M. G. H. Coles, \& G. D. Logan (Eds.), Converging operations in the study of visual selective attention (pp. 395-414). Washington, DC: American Psychological Association.

LAVIE, N., \& Driver, J. (1996). On the spatial extent of attention in object-based visual selection. Perception \& Psychophysics, 58, 1238-1251. 
Loftus, G. R., \& Masson, M. E. J. (1994). Using confidence intervals in within-subject designs. Psychonomic Bulletin \& Review, 1, 476-490. MARR, D. (1982). Vision. San Francisco: Freeman.

Navon, D. (1977). Forest before trees: The precedence of global features in visual perception. Cognitive Psychology, 9, 353-383.

Palmer, S. E. (1977). Hierarchical structure in perceptual representation. Cognitive Psychology, 9, 441-474.

POSNER, M. I. (1980). Orienting of attention. Quarterly Journal of Experimental Psychology, 32A, 3-25.

Stevens, K. A., \& Brookes, A. (1988). The concave cusp as a determiner of figure-ground. Perception, 17, 35-42.

TARR, M. J., \& PINKER, S. (1989). Mental rotation and orientationdependence in shape recognition. Cognitive Psychology, 21, 233-282.

TARR, M. J., \& PINkER, S. (1990). When does human object recognition use a viewer-centered reference frame? Psychological Science, 1, 253-256.
VeCERA, S. P. (1994). Grouped locations and object-based attention: Comment on Egly, Driver, and Rafal (1994). Journal of Experimental Psychology: General, 123, 316-320

Vecera, S. P., \& Behrmann, M., \& Filapek, J. C. (in press). Attending to the parts of a single object: Part-based selection limitations. Perception \& Psychophysics.

Vecera, S. P., \& FARAH, M. J. (1994). Does visual attention select objects or locations? Journal of Experimental Psychology: General, 123, 146-160.

WATT, R. J. (1988). Visual processing: Computational, psychophysical, and cognitive research. Hove, U.K.: Erlbaum.

(Manuscript received January 12, 1999; revision accepted for publication June $30,1999$. 